NASA TECHNICAL NOTE

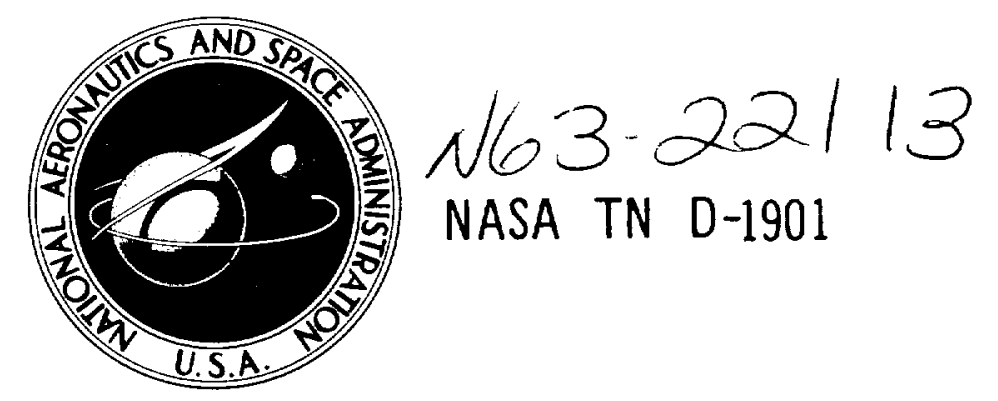

$\overrightarrow{8}$
$\frac{9}{1}$
$z$
$i$
$\frac{1}{2}$
$\frac{1}{2}$

\title{
THE PLASMA IN INTERPLANETARY SPACE
}

by L. Biermann

Max Planck Institute for Astrophysics

Munich, Germany'

NATIONAL AERONAUTICS AND SPACE ADMINISTRATION • WASHINGTON, D.C. - OCTOBER 1963 

TECHNICAL NOTE D-1901

THE PLASMA IN INTERPLANETARY SPACE

By L. Biermann

Max Planck Institute for Astrophysics

Munich, Germany

NATIONAL AERONAUTICS AND SPACE ADMINISTRATION 



\title{
THE PLASMA IN INTERPLANETARY SPACE
}

\author{
by \\ L. Biermann \\ Max Planck Institute for Astrophysics
}

\section{SUMMARY}

Interplanetary plasma, including the various lines of evidence for it, is described. This is followed by an account of the structure and composition of comets with emphasis on the ionized tails. The characteristics of these tails are explained by their interaction with a "solar wind" type plasma. Cometary phenomena bearing on the spatial and temporal distribution and dynamics of the plasma are discussed. A space experiment consisting of the formation of an artificial plasma cloud whereby these ideas could be tested and the plasma further investigated is described. Calculations indicate that the release of about $100 \mathrm{grams}$ of barium at $200,000 \mathrm{~km}$ could be observed. 



\section{CONTENTS}

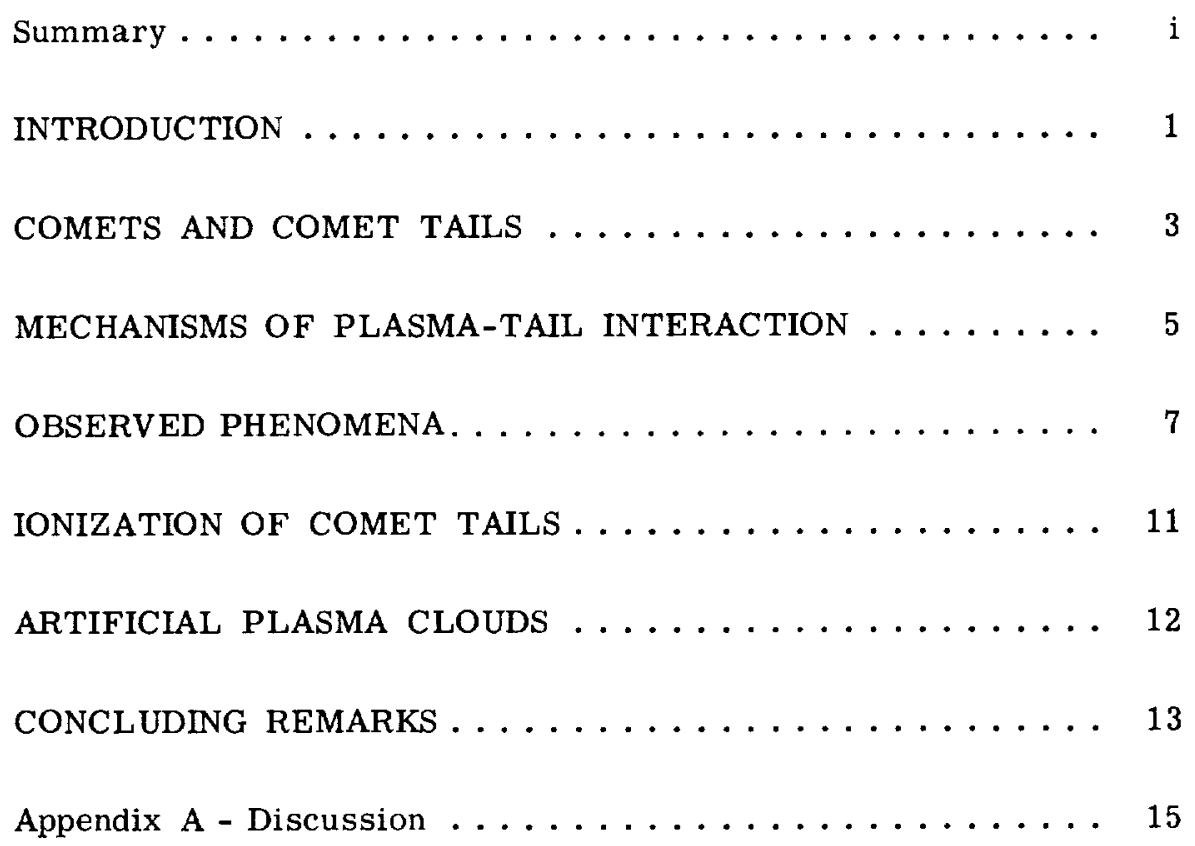





\title{
THE PLASMA IN INTERPLANETARY SPACE*
}

\author{
by \\ L. Biermann ${ }^{\dagger}$ \\ Max Planck Institute for Astrophysics
}

\section{INTRODUCTION}

The subject of plasma in interplanetary space has been attacked by astronomical observation and theoretical work for a number of years. It is gratifying therefore that experimental research on it has now become possible by means of space probes and satellites with distant apogees.

Interplanetary space can be defined literally as the space between the sun and the planets. There has been some discussion in recent years as to what extent it should be regarded as an extension of the solar corona. We shall not, at present, go into the problem of how to define the boundary between the solar corona and interplanetary space. Let us just take the position that the corona, as is suggested by its appearance during a total solar eclipse, stretches outward to about 10 and possibly 20 solar radii, and that beyond this distance we reach interplanetary space.

The question of the physical distinction between the solar corona and interplanetary space is rather difficult. Leaving aside the radial motion, there is one basic difference as far as the corotation with the sun is concerned. The visible corona does co-rotate with the sun; this means that it rotates once in every 25 days. From the work to be discussed here, it is evident that interplanetary material, at distances of the order of $1 \mathrm{AU}$, does not co-rotate. One of the tasks before us, then, is to determine in detail the state of rotation of the material found in interplanetary space, as a function of position and time. This question cannot as yet be answered from observation.

How is plasma in interplanetary space observed? In addition to the indirect evidence from magnetic storms (see below) and the recent direct measurements there is first the body of information from the comet tails. Comets will be discussed later, in more detail; let us just say now that there is one type of tail, which is-by convention-called a Type I tail. These tails are essentially straight and long, directed approximately radially away from the sun, and are found to consist of ions of $\mathrm{CO}$ and $\mathrm{N}_{2}$ and of some other ionized molecules in overall densities ranging from a few to perhaps $10^{2}$ or $10^{3}$ particles $/ \mathrm{cm}^{3}$; however there are usually structures in which the density is considerably higher; and the density also varies with distance from the head. From plasma physics we know that

\footnotetext{
* Presented at a NASA Goddard Space Flight Center Colloquium on October 17, 1961, Greenbelt, Maryland. This talk together with the question-answer period which followed (Appendix $A$ ) is published here in approximately the form of the original presentation; for this reason references to other work in the field are presented as originally given.

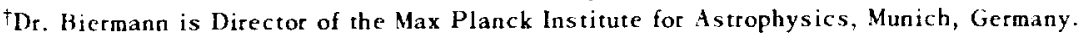


this material must necessarily constitute a quasi-neutral gas; that is, there must be as many electrons per cubic centimeter as there are ions. These molecular ions move away from the sun at velocities on the order of 30 up to several hundred kilometers per second. This is the kind of plasma that for several decades has been known from observation to exist in interplanetary space and in the place where there is a comet with a Type I tail.

For a large comet, the statistical probability of having a Type I tail is something like 50 percent; this estimate is based on evidence taken from a total of more than 50 comets [This is mostly from the work of Bredichin-Jaegermann (Petersburg 1903) as discussed by P. Stumpff, Astr. Nachr. 286:87 (1960). A new discussion on the basis of a very much larger material has been started.]

Second, there are the direct measurements of the interplanetary plasma from satellites or space probes; the most significant work in this area has been done recently by Bridge, Rossi, and their coworkers at the Massachusetts Institute of Technology. Some important work has been done also in Russia; this, in a general way, confirms the MIT results but doesn't give as many details.

At this stage it is sufficient to say that, during geomagnetically quiet periods, in the vicinity of the earth (at a distance of approximately $200,000 \mathrm{~km}$ ) there are densities of the order of 10 ions (and electrons) per cubic centimeter; these ions move radially away from the sun with velocities on the order of $300 \mathrm{~km} / \mathrm{sec}$.

Third, we have evidence from the observation of radio sources near the sun. If by the apparent motion of the sun along the ecliptic during the year a radio source happens to lie on a line passing near the sun, say within 50 or 100 solar radii (equivalent to 12 to 25 degrees of arc) from the sun, it has been observed that the apparent size of the radio source is affected in a way that indicates the influence of some sort of scattering.

A more detailed discussion shows that there must be scattering by electrons in interplanetary space. While nothing can be derived about their state of motion, some rather indirect information may be gained about the density distribution.

Fourth, the observation of zodiacal light yields some evidence. Quite a number of investigations on this subject have been reported in the literature; but much of the earlier interpretation of the data has been superseded by later research.

The only reasonably certain conclusion at the present time is that during magnetic storms there might be electron densities of the order of some $10^{2}$ electrons $/ \mathrm{cm}^{3}$.

There is also the general evidence from geomagnetism. Chapman and Ferraro have shown how magnetic storms are caused by plasma streams emitted from the sun and moving through interplanetary space with a velocity on the order of $1000 \mathrm{~km} / \mathrm{sec}$. But evidence as to the densities of these plasma streams is rather meager, and it is very difficult to give more than a lower limit.

Also, from geomagnetic evidence, two types of events should be distinguished. First, the magnetic storms: These are individuals events often connected with chromospheric eruptions on the sun. Second, there are recurrent streams-usually phenomena of a smaller scale-that have a tendency to 
repeat themselves after 27 days, possibly three times, four times, or even more often. These are presumably due to persistent regions on the sun called M-regions, which are believed to be semipermanent sources of rather intense corpuscular emissions, their lifetime being on the order of several months or more.

The question of whether-in addition to the individual big events and the recurrent but quasistationary emission-there is also a stationary, more or less permanent and roughly isotropic outflow of coronal gas cannot be answered positively from geomagnetism. There has been a growing tendency in geomagnetism to connect the fluctuations of the geomagnetic field at polar stations, which are practically always observed, with matter coming from the sun. It may be expected that the observations made during the IGY will settle this question.

The main question that we will discuss today is this: Excepting the Type I tails of comets, the isolated solar eruptions that cause magnetic storms, and the recurrent streams of solar matter, is there always some plasma moving through interplanetary space? What we hope to show is that the observations of the comets' tails do indeed give a rather strong reason to believe that there is practically always some outflow of ionized matter from the sun.

\section{COMETS AND COMET TAILS}

The evidence from the comets and the interpretation of the acceleration and ionization of the cometary material is now given somewhat more in detail. Also, some plans for using the processes in the comet's tails as a model for an experiment will be discussed briefly. For this experiment, artificial plasma clouds ? re to be produced in interplanetary space. These should be directly observable and should behave in a way similar to the comets' plasma tails-and serve as probes for the presence of interplanetary material and its state of motion.

As a background to this discussion, a brief description of a comet will be helpful. First, a comet is believed to have a solid nucleus with a diameter, say, of a few to 10 or 20 kilometers, which consists of dust and of molecules of $\mathrm{C}, \mathrm{N}, \mathrm{O}$, and $\mathrm{H}$ in frozen form. There is good reason to assume that all this material gives rise to the appearance of the comet during its near approach to the sun, when this more or less solid nucleus is under the influence of solar light and heat. The nucleus is not very solid in the sense that the molecules present probably form a sort of ice that is frozen together with the dust; this model of a cometary nucleus has been developed and discussed particularly by Whipple at the Smithsonian Institute.

If a comet approaches the sun to within a few astronomical units, it gets a coma consisting of nonionized gaseous molecules like $\mathrm{CN}, \mathrm{C}_{2}, \mathrm{C}_{3}, \mathrm{CH}, \mathrm{NH}$, and $\mathrm{OH}$, and in addition one or two tails. In the latter there are dust particles and/or ionized moledules like $\mathrm{CO}^{+}$and $\mathrm{N}_{2}^{+}$. The connection between composition and the type of the tail is this: The long, straight Type I tails are composed of ions, and other more curved types of tails are composed of dust and possibly some nonionized molecules. But here we shall discuss mainly the Type I tails.

Around the solid nucleus of the comet is the coma, very roughly 100,000 to a million kilometers in diameter. Furthermore, we may have a straight, extended tail that would be of the Type I and/or a shorter, curved tail that would be of Type II or III. These latter two types need not be distinguished 
for our purpose, but it should be noted that their direction with respect to the sun is usually different from that of the Type I tail. Type I tails are the longest, their length being of the order of about 10 million kilometers, and in some cases even more.

Both the dust and the nonionized molecules are subject only to a moderate or small acceleration away from the sun, whereas the ionized particles are driven away from the sun much faster. The former show accelerations away from the sun that are comparable only to the acceleration of gravity $\left(0.6 \mathrm{~cm} / \mathrm{sec}^{2}\right.$ at $\left.1 \mathrm{AU}\right)$. In contrast, the acceleration acting on the constituents of Type I tails-that is to say on the ions-is very much larger, say by a factor between 20 and 100 . In individual cases it appears to go up to $1000 \mathrm{~cm} / \mathrm{sec}^{2}$, and even more.

This general picture explains the difference of the shapes. The motion of particles under the influence of solar gravitation is essentially a problem of celestial mechanics that was solved by astronomers during the last century. Thus one could explain, in a general way, the shapes of the tails of the Type II and III-and in less detail also the shape of the Type I tails.

With regard to the mechanism of acceleration away from the sun, it should be added that the pressure of the ordinary solar light is quite sufficient to account for the observed features of the tails of Type II or III. For dust particles that are small enough, the acceleration by light pressure is of the right order of magnitude; for nonionized molecules we may readily compute the light pressure from the transition probabilities in question, and again the acceleration turns out to be on the order of unity. Hence there is no particular problem in accounting for the observed acceleration of the nonionized molecules and of the dust particles.

There is, however, a serious problem concerning the ionized particles, because the transition probabilities of the resonance transitions in question in this case are quite small. The best value for the oscillator strength of these transitions is only 0.002 . That means that the light pressure on these ions can account only for something like $0.1 \mathrm{~cm} / \mathrm{sec}^{2}$. So, if the light pressure were operative, we would expect an acceleration that would be smaller by about one power of 10 as compared with the $\mathrm{CN}$ or $\mathrm{C}_{2}$. What we observe in fact is that it is very much larger.

First, some of the main properties of these Type I tails should be mentioned. They are approximately parallel to the radius vector from the sun, as was stated before. In the second approximation there is a phenomenon of quite some interest: It was found first by Hoffmeister that the main axis of the comet deviates slightly from the radius vector so that the tail is lagging behind, in the sense of the comet's motion around the sun, by a few degrees of arc-say, by something like 3 to 5 or 6 degrees. This is significant from the theoretical point of view, which we shall develop later.

Next we have the extent of these tails, mentioned already. While the diameter of the tail is on the order of some $10^{5}$ to $10^{6} \mathrm{~km}$, which is comparable to the diameter of the coma, the length may be very much larger. There have been a few comets possessing tails that extended even through a large part of the solar system, up to distances comparable with the distance between the earth and the sun. 
There are usually structures in the tails of Type I. For example, there are elements that might be described as clouds and that can easily be followed on successive plates from one hour to the next-and sometimes from one night to the next, or even longer.

Velocities can be derived by measuring structures, e.g., "clouds" appearing on successive photographic plates. The values are of the order of 20 or 30 to $50 \mathrm{~km} / \mathrm{sec}$, but sometimes are several hundred to $1000 \mathrm{~km} / \mathrm{sec}$. The ordinary range, however, is 30 or 50 to $200 \mathrm{~km} / \mathrm{sec}$.

An average value of the acceleration can also be found by plotting the velocity as a function of the distance. The time scale of the motion of an individual element (that is, the time during which we may say that a given element of mass is visible in the comet itself) is of the order of some days. This means, furthermore, that the tail is a transient phenomenon; for while the time of visibility of every comet is of the order of, say, several months-depending on the perihelion distance and the other conditions, the material that can be seen one night in the tail is replaced for the most part after a week, and certainly completely so after a fortnight.

Another interesting and frequent phenomenon is presented by very narrow streamers, a regular feature of many tails. These are structures with a diameter of only a few thousand kilometers and a length of the order of $10^{6} \mathrm{~km}$. The fact that they are so narrow is again significant: If the material would expand sideways with the velocity of sound, then a very low temperature of the order (of a few degrees Kelvin) would be required to keep it that narrow for the length of time given by the extent and the velocity. So there must be some lateral force that confines the streamers; and the only likely explanation is that there are magnetic fields associated with the streamers which, by their pressure, keep the plasma together.

A particularly important feature of these tails is what might be termed their activity. If several successive pictures of a Type I tail are compared, the variations from one plate to the next are usually quite conspicuous: Part of the material is moving farther away from the coma, the shape is changing, and material that has been at some distance from the axis of the tail has often come nearer to it.

If we compare pictures taken during successive nights, then we see that the whole appearance of the tail usually has changed considerably. Part of the tail material has been largely replenished, and at the same time there has been considerable displacement and rearrangement. A structure seen on one side of the tail axis during one night may even have moved to the opposite side of the axis on the next night; there may be in such cases, in addition to the displacement along the axis, a rotational motion around it. The plates of the comet Mrkos give an example of this phenomenon.

\section{MECHANISMS OF PLASMA-TAIL INTERACTION}

Briefly stated, the main point of the hypothesis that was introduced just 10 years ago is as follows.

The acceleration of the plasma, which forms the Type I tails, and the "activity" of these tails are attributed to the influence of solar particle radiation. The large acceleration is ascribed to the 
fact that, from plasma physics, the interaction between two interpenetrating streams of plasma is known to be large, especially in the presence of magnetic fields. The tail plasma is moving, at least initially, quite slowly as compared with the particle streams coming from the sun. We would expect on quite general grounds that there will be a coupling between these two plasmas.

One basic consideration is that, if we ascribe the acceleration of these tails to the influence of solar corpuscular radiation, then their activity is a consequence of solar activity. Ordinary solar light is constant within a fraction of 1 percent. So we would not expect any variation of the acceleration because of the pressure of solar light acting on the structures composed of nonionized molecules and of dust.

On the other hand we know from geophysics that the particle radiation of the sun shows a great deal of variation, even between magnetic storms, and at a generally low level of solar activity; so it is natural to assume that the activity of the comet's plasma tails just reflects this type of activity of the sun-the activity which shows up on the earth as magnetic activity and in various other geophysical phenomena.

Now, in order to give substance to this hypothesis, let us first check whether the momentum flow coming from the sun is really sufficient. Instead of the line of reasoning that was current some years ago, the evidence from the measurements of Bridge, which give perhaps a lower limit to the particle flux, will be used. If we take the proton density and the velocity that Bridge measured-the latter on the order of several hundred kilometers per second, we get a momentum flow from the sun under quiet solar conditions of $10^{-8} \mathrm{dyne} / \mathrm{cm}^{2}$.

Again, if we take average figures for a Type I tail at some distance from the head and the lower velocities observed in these tails under quiet conditions, we get values on the order of some $10^{-9}$ dyne $/ \mathrm{cm}^{2}$.

We know that the momentum flow from the sun is very much increased in the recurrent streams observed during low levels of solar activity or during magnetic storms. It is somewhat difficult to write down a precise figure, but all evidence points to increases in the momentum flux of up to several powers of 10 . These, of course, would show up in the comet's tail by more violent phenomena.

The momentum flow, then, seems adequate to accelerate the comet's tail provided the coupling between the tail plasma and the solar plasma is sufficiently strong.

With regard to the mechanism of coupling, there are three possibilities. One is that coupling is due merely to the thermal motion of the electrons; this scheme was discussed in detail in 1951 . It can be shown to be operative if the density of the solar stream is on the order of $10^{2}$ or $10^{3} / \mathrm{cm}^{3}$ and if the electron temperature is on the order of $10,000^{\circ} \mathrm{K}$. While in 1951 there was some observational evidence pointing toward densities in this range, we believe now that these are not there under average conditions.

Second, there is the possibility also discussed 10 years ago, and rediscussed by several authors since then, that magnetic fields couple together the solar plasma and the plasma of the comet's tail. This possibility appeared fairly remote in 1951, but in the meantime we have much indirect evidence 
about the presence of magnetic fields in interplanetary space; furthermore there are the direct measurements by means of space probes or satellites, such as those by Dr. Heppner. In addition, there is the evidence from the very fine streamers which, as was noted, give strong reason for believing that there are often (perhaps regularly) magnetic fields in the Type I tails. We may ask, then, whether the magnetic stresses are large enough to account for the observed transfer of momentum.

Since the magnetic fields that have been observed directly under quiet conditions are on the order of 10 to 20 gamma ( 1 to $2 \times 10^{-4}$ gauss), evidently there are magnetic stresses on the order of $10^{-9}$, or several times $10^{-9} \mathrm{dyne} / \mathrm{cm}^{2}$; this seems adequate to account for the observed momentum flux.

Third, it might be that specific plasma effects due to plasma instabilities, such as fluctuations of the electric fields, are involved here. This is a possibility that has become apparent recently in connection with the experiments in plasma physics related to the problem of controlled fusion.

The state of our knowledge in this area is not yet sufficient to allow a definite statement as to whether or not this type of plasma coupling should effectively increase the coupling between a solar particle stream and cometary plasma.

\section{OBSERVED PHENOMENA}

Let us now consider observational effects that might, or might not, support this picture.

First of all, of course we have to ask whether we find correlations or recurrence phenomena (due to the sun's rotation) similar to those known in geomagnetism. If, for instance, we have a situation in which the earth and the comet are (in this order) approximately along one radius vector from the sun, then we would expect that, whenever we observe a really big magnetic storm on the earth, we should also observe something spectacular happening in the comet's tail.

That is to say, we should expect a close correlation between geomagnetic phenomena and the phenomena in the comet's plasma tail. Of course, the condition described above does not happen often. But there have been some such cases-for instance, the comet 1942g, Whipple-Fedtke; the comet was moving approximately in the plane of the ecliptic, and furthermore the perihelion of the comet coincided approximately with the opposition to the sun as seen from the earth. This is another favorable geometrical condition since, when the comet is in perihelion, the earth might be in any longitude; so it is really by chance that they both are in approximately the same heliocentric longitude. As a consequence, the comet could be seen in approximately the same direction of the sky (relative to the sun) for a fairly long time.

This was a time during which there were only a few magnetic storms. There was one particular storm, the largest for about one-half year, on March 29, 1943, which showed up in the comet's tail by an exceptionally large acceleration.

Good observations of this comet were obtained at the Sonneberg Observatory. Values of the acceleration were derived for a number of individual days by the method that we described before. 
There are two dates in 1943 where the motions in the tail were exceptionally fast and turbulent-March 3 and March 29. (The comet had been found late in 1942, but observations were mainly obtained during the first months in 1943.) Now it can be seen that the interval between these two dates is approximately one period of the sun's rotation.

Here the synodic period of rotation of the sun depends on the motion of the body to which it is referred. The sidereal period-that is, the true period of rotation of the sun-is 25 days. But, since the earth is moving in the same sense as the sun rotates, the sun appears to rotate only with a period of 27 days. For the comet Whipple-Fedtke, it would have been 26.5 days. Thus, this is the recurrence period to be expected if the high degree of turbulence observed on March 3rd and 29th was due to the corpuscular radiation emitted by the same active region on the sun. As has been noted, on one of these two dates (March 29th) there was a major magnetic storm on the earth that showed up very drastically in the structure of the tail. In contrast, on March 3rd the event in the tail of the comet was not accompanied by a particular event in the geomagnetic records but there was another geomagnetic event 27 days after March 29th. Of course, there was always some angle (20 to 30 degrees) between the comet and the earth, as seen from the sun; it is known that the streams coming from the sun have only an angle of opening of the same order.

In consequence, the observed correlation with the geomagnetic storm of March 29th and the apparent recurrence period of 26 days is probably just what we should expect under these circumstances. [See the pictures reproduced in $Z$. f. Astrophys. 54:67 (1962) and 22:286 (1943), especially pp. 304, 305.]

Another investigation was made for the comet Halley as observed during its reappearance in the year 1910. Again it was possible in observing the comet to find a synodic period of rotation of the sun as seen from the comet Halley. In that case it was only 22 days, because the comet Halley moves around the sun in a direction opposite that of the earth; therefore, the value is less than 25 days. Also, it was possible, by an appropriate analysis, to get some correlation with geomagnetic phenomena; however, this was somewhat more difficult because the general activity of the sun was at a higher level than 1943. Suffice it to say that the general result was again in agreement with that anticipated.

There are a few more events of this type that have been discussed, mainly by Rhea Lüst in some recent papers. The expected correlation between cometary and geomagnetic events and recurrence phenomena in analogy to those known in geomagnetism thus have been found in the plasma tails of the comets.

Furthermore, as mentioned before, the tail in general appears to lag behind the radius vector from the sun by a small angle, in the sense of the comet's orbital motion. That, again, is precisely what we would expect in case of a mechanical transfer of momentum if the material is coming from the sun with a speed of, say, approximately 10 to 20 times the orbital velocity of the comet-that is, a few hundred kilometers per second.

If instead we have electromagnetic coupling, the situation is somewhat more complicated; but from the momentum balance we would still expect an effect of this general character. So we may take the observation of this angle as another qualitative verification of the basic idea. 
If, then, we regard the hypothesis that the acceleration of the plasma tails of comets is due to the interaction with the solar corpuscular radiation as verified, we can immediately draw one or two conclusions not obvious from geomagnetism. The first is connected with the fact that if a comet is once observed to have a plasma tail, then it is found to possess such a tail, though with varying properties, subsequently during the whole time of its near-approach to the sun. The tail is ordinarily visible as long as the distance from the sun is smaller than about $3 / 2 \mathrm{AU}$. That is to say, if a comet gets this type of tail at all, it tends to have it for the whole time it is inside (approximately) the orbit of Mars.

But, as we have said, of the brighter comets only one in approximately two does get an observable plasma (Type I) tail; presumably the property of getting such a tail depends on the chemical composition and possibly also on the past history of the comet.

It should perhaps be mentioned here that a given comet may make something like 100 nearapproaches to the sun. There are about $10^{11}$ potential comets in the sky, most of which are moving at distances almost comparable with the distances of the nearest stars. All these bodies move through interstellar space with the sun and are hence actually members of the solar system. But, through the gravitational disturbances caused by stars passing nearby, their constants of motion are changed statistically; and so it happens that one or two every year are thrown into orbits that touch the inner part of the planetary system. As soon as they approach the orbit of Jupiter or Saturn, their constants of motion are changed in such a way that they either disappear entirely (by getting into an hyperbolic orbit around the sun) or, with equal probability, they get into an orbit having a major axis of the order of 20 or $30 \mathrm{AU}$ (e.g., comet Halley).

From then onward the comet may be in this orbit for about 50, 100, or 200 revoltuions around the sun. But the nucleus, losing something like 1 percent of its material in each approach to the sun, is not a permanent body. In a few cases we have witnessed the breaking up of a comet during its near-approach to the sun.

It has been mentioned that a comet with a Type I tail appears to have this tail during most of the time spent in the inner part of the planetary system. That means, then, that the solar corpuscular radiation is not there exclusively during the time of magnetic storms or recurrent magnetic phenomena but that there must be some corpuscular radiation all the time; otherwise, we would have to assume a quite unlikely correlation between the appearance of comets and the production of solar corpuscular radiation. For this component of the solar corpuscular radiation, Eugene Parker has proposed the term "solar wind"; this seems a very appropriate term as long as it is not used also for the more violent phenomena of recurrent character or of the magnetic storm type.

We observe furthermore that comets with Type I tails are not confined to periods of high solar activity. They are actually rather scattered through the whole 11-year cycle, although there may be some dependence on phase in this cycle as a second-order effect. Out of the total number of something like 30 comets with Type I tails which have been looked at more closely so far, it is not possible to give a more precise estimate*.

"See also R. Lüst, 7. f. Astrophysik 51:163 (1961) and 57:192 (1963). 
The comets are moving around the sun not only in the neighborhood of the plane of the ecliptic, but in orbits that may have any inclination to that plane: There have been comets that passed the vicinity of the poles. We might therefore wonder whether there is any difference in the properties of these comets as compared with those moving near the ecliptic plane.

A preliminary study of this kind has been made by Peter Stumpff [Astron. Nachrichten 286:87 (1961)]. He separated the comets for which enough data were available into several groups according to the heliographic position of their perihelion.

Dividing the sky into two areas of equal angular area-one centered around the ecliptic plane (approximately the equatorial plane of the sun) and the other half around the poles, Stumpff found that the ratio of comets with plasma tails to the comets without such tails differed by a factor of 2 . The overall probability of a comet having a plasma tail seems to be something like $2 / 3$ if its perihelion is within 30 degrees of the ecliptic plane as compared to about $1 / 3$ if its perihelion is within 60 degrees of the poles.

This result appears to be consistent with evidence from the occultation of radio sources, which again gives the impression that the scattering electrons in interplanetary space have nonspherical surfaces of equal density around the sun; these observation indicate some flattening of these surfaces out to 60 to 100 solar radii, but not at all an absence of scattering electrons at high latitudes. The electrons in question should, of course, belong to the solar corpuscular radiation.

There is another conclusion: We observe that the momentum transfer from the solar particle streams to the plasma of the comet is to the first order of approximation along the radius vector from the sun; to the second order we have definite indication that the individual motion of the comet plays some role. This means that velocities on the order of 20 or $30 \mathrm{~km} / \mathrm{sec}$ perpendicular to the radius vector really do show up in the general direction of the tail.

This indicates now that the material coming from the sun is not coupled to the sun in the sense that it would co-rotate. Complete co-rotation with the sun would mean that the material near the earth would have a velocity on the order of $450 \mathrm{~km} / \mathrm{sec}$. If the material emitted as corpuscular radiation from the sun would co-rotate at the earth's distance, we should observe a speed perpendicular to the radius of the order of 400 to $500 \mathrm{~km} / \mathrm{sec}$.

The fact that we are able to observe the second-order effect reflecting the individual motion of the comet, which is something like one-tenth or less than this, indicates that there is no co-rotation of the interplanetary plasma within, say, $20 \mathrm{~km} / \mathrm{sec}$ or so.

This is mentioned specifically because it was proposed some time ago that the solar time variation of the intensity of cosmic radiation be ascribed to a co-rotation with the sun of the interplanetary plasma in the vicinity of the earth. It would seem that the evidence from the comets quite definitely rules out this hypothesis.

Of course, that leaves us with this question: Out to what distance do we have co-rotation with the sun? If we put together all the data on the electron density in interplanetary space as a function of the distance from the sun, we don't find any discontinuity. Of course, there are certain arguments 
discussed by R. Lüst, and by Lüst and Schlüter, which tend to show that, say, in the vicinity of the orbit of Mercury there is a surface beyond which there is no co-rotation and inside of which there may possibly be complete co-rotation as observed in the solar corona. This surface may be at a distance of, say, $1 / 3 \mathrm{AU}$; but this estimate is highly uncertain. This is mentioned only as one of the questions that certainly should be answered by means of observations from space probes. One possibility would be to produce artificial plasma clouds much nearer to the sun and to make observations from a position that would permit us to see what is happening to them.

\section{IONIZATION OF COMET TAILS}

Another problem connected with these (Type I) plasma tails-the problem of the ionizationshould be discussed briefly.

We mentioned the observable constituents, ions like $\mathrm{CO}^{+}$and $\mathrm{N}_{2}^{+}$and some others: All of these have ionization potentials of the order of 14 or so electron volts.

Using Hinteregger's data to derive the time scale of photoionization, we get a result of the order of one to several months. That, of course, is by far too long to account for the observed rate of appearance of ion structures; therefore, if the best measurements known at present can be trusted, the ionization of these ions cannot be due mainly to the photoionization of the parent molecules (CO, $\mathrm{N}_{2} \ldots$...

That, then, leaves us with the problem of how we can account for the ionization, especially for the rapidity with which it sometimes occurs. Two or three possibilities will be mentioned.

The one discussed in the literature, in addition to photoionization, is the process known in atomic physics as exchange of charge. That is to say, a solar proton, in encountering a nonionized CO or $\mathrm{N}_{2}$ molecule, may produce an ion of $\mathrm{CO}$ and becomes itself a neutral hydrogen atom; this happens with a large cross section if the velocity is in range of, say, 200 to $2000 \mathrm{~km} / \mathrm{sec}$. It has been found experimentally that the cross section depends strongly on the relative velocity. For the velocity range in question it is around $3 \times 10^{-15} \mathrm{~cm}^{2}$, a relatively large cross section.

The time scale of the order of 1 day (approximately $10^{5}$ seconds) for the formation of ionized structures would then require a particle flux of $10^{9.5}$ protons $/ \mathrm{cm}^{2}-\mathrm{sec}$, provided the charge exchange takes place as in free interplanetary space.

Until a short time ago that seemed to be a satisfactory explanation. A particle flux of $3 \times 10^{9}$ protons $/ \mathrm{cm}^{2}-\mathrm{sec}$ would correspond, say, to 50 protons $/ \mathrm{cm}^{3}$ moving with a velocity of $600 \mathrm{~km} / \mathrm{sec}$. These figures were compatible with all the evidence available until about last year. The upper limits given by other means are around 100 particles $/ \mathrm{cm}^{3}$ for quiet conditions.

Now the measurements of Bridge and the similar experiments by the Russian physicists tend to indicate a flux of solar materials under quiet conditions of only several times $10^{8}$ protons $/ \mathrm{cm}^{2}-\mathrm{sec}$. This would correspond to a particle density on the order of $10 / \mathrm{cm}^{3}$, and to a velocity on the order of, say, $300 \mathrm{~km} / \mathrm{sec}$ and would leave for the flux a discrepancy by a factor of around 10. 
Occasional observations indicate that the time scale of the formation of ion structures is as short as 1 hour; this means that we would have to have another $3 / 2$ powers of 10 . But these last measurements are probably characteristic for disturbed conditions, that is, to higher solar activity.

To account for the ionization, there are two possibilities in addition to charge exchange mentioned in current discussions or in the literature: One is ionization by electron collisions, while the other one is a binary chemical reaction of the following type: starting, say, with $\mathrm{C}^{+}+\mathrm{O}_{2} \longrightarrow \mathrm{CO}^{+}+\mathrm{O}$, which is both exothermic and has a relatively large cross section.

With regard to electron collisions, there is the difficulty that we need electrons with energy of at least something like $25 \mathrm{ev}$ because the cross section for ionization is quite low for electrons with smaller energy.

With such electron energies we would expect, first, collisional dissociation rather than ionization, with quite a number of competing processes in part of which negative ions are formed. So it would seem that the chances that electron collisions contribute efficiently to ionization are not very good.

The other possibility has some slight advantages: The ionization potential of $\mathrm{C}$ is only $11.63 \mathrm{ev}$; hence the photoionization should be somewhat faster than that of CO.

The ionization potentials of $\mathrm{CN}$ or $\mathrm{C}_{2}$ are not known, but we might guess that they would be similar to that of $\mathrm{C}$. Unfortunately the photoionization cross sections for these are, as far as we know, not yet available. Summing up, it may be said that the cause of the ionization could be complex although, on the balance, the mechanism of charge transfer is likely to be the most efficient contributor.

\section{ARTIFICIAL PLASMA CLOUDS}

Lastly, a few words should be said about the possibility and usefulness of producing artificial plasma clouds in interplanetary space to obtain more information about the properties of solar corpuscular radiation by direct observation.

Evidently what we would like to have is a material that, in ionized form, has at least one fairly strong transition in the ordinary optical range and at the same time becomes photoionized by ordinary solar light as rapidly as possible.

If we look at the periodic table we find easily that, practically, only some of the alkali earth atoms are really useful.

Next we inquire what quantities are needed in order to enable us to observe such clouds reasonably well without extra techniques, such as special filters or image converters-which of course would considerably increase the possibilities of this type of work.

With this narrow restriction, we have worked out the question in some detail. However, we will not go into this, since a paper containing all this information will appear quite soon in the Zeitschrift für Astrophysik (see vol. 53, p. 226). 
The minimum final figures needed for an assumed cloud distance of 200,000 km are: for calcium, $2 \mathrm{~kg}$; for strontium, $6 \mathrm{~kg}$; while, for barium, only $130 \mathrm{gm}$.

The reason that we require a rather large quantity of calcuim is that the main calcium resonance absorption line is quite strong in the solar spectrum itself.

Also, the strontium line is at a disadvantage by its position in the solar spectrum; only the barium resonance doublet (around 4600 and $4900 \mathrm{~A}$ ) is really in a very good region of the solar spectrum. This and the fact that the photoacceleration of the barium ion is not large $\left(6 \mathrm{~cm} / \mathrm{sec}^{2}\right)$ overbalance the disadvantage that barium has the largest atomic weight (135).

So the general technique would be to release a quantity of barium gas (approximately $100 \mathrm{gm}$ or more at a distance of $200,000 \mathrm{~km}$ ) and to see how the solar plasma interacts with it. This would give information directly about the direction of acceleration; it would also give indirect information about the mechanism of coupling if we use, say, a mixture of barium and calcium, where the large difference in atomic weights might discriminate between mechanisms. Such a difference would help us to distinguish between, for instance, plasma acceleration and the influence of magnetic fields.

The sideways spreading of the material should give information on the interplanetary magnetic fields, but these details will not be discussed here.

In deriving the figures given so far, it was assumed that the surface brightness of the cloud should be greater than the integral surface brightness of the sky-a condition that would have to be fulfilled when ordinary observing techniques are used. But, by applying a filter, we should gain some advantage.

With techniques of this sort, we could furthermore hope to use not only alkali earths but also carbon monoxide-but only during periods of very much enhanced solar particle flux. If we would make such an experiment during a big magnetic storm, we should indeed be able to observe directly the ionization of the $\mathrm{CO}$ by exchange of charge. Since we know the cross section and we know precisely the amount that we have released, we should get the time scale of the ionization just by watching the appearance of this carbon monoxide ion. Knowing the quantity involved, we should be able to make an independent quantitative check of the intensity of the solar particle stream.

This, of course, is only of interest if the experimental measurements with plasma probes appear to be so difficult that we would like to have a check done by an entirely independent method. If, on the other hand, we are confident that the available experimental techniques are perfectly adequate, then perhaps there is no particular point in making an experiment with $\mathrm{CO}$ since it might not be regarded as worthwhile from our interest in the physics of comets.

\section{CONCLUDING REMARKS}

Let us conclude with one general remark. To the extent we succeed in understanding what is going on in the comets, specifically in the plasma tails of comets, we can use these tails as probes provided by nature. That has, of course, an advantage, because then we can use all the past 
observations of comets which have appeared in the literature. We mentioned the comets moving above the poles of the sun and the fact that they appear in all phases of the solar cycle. So, in spite of the fact that we are fairly confident in the near future of a number of space probes and very distant satellites to give us direct measurements, it might still be worthwhile to have additional clues from this very rich material in the form of past observations of comets. These data certainly would give us information of a somewhat different character-for instance, if we inquire about statistical properties of solar corpuscular radiation over long periods of time, or its properties at high heliographic latitudes, or in a part of the solar cycle for which we shall now have to wait 10 years. In this connection the intended experiment of Dr. Donn of Goddard Space Flight Center is very much welcomed, although its aim is somewhat different from that of our own experiment. It should help us also to understand more in detail the processes which take place on comets, and thus, though perhaps somewhat more indirectly, assist us in using the comets as probes for the interplanetary plasma. 
Appendix A

\section{Discussion}

QUESTION: As you undoubtedly know, the Russians injected a couple of plasma clouds at fairly large distances from the earth. These were observed for periods of several minutes which, judging from the acceleration you gave, might-the motion, say, due to solar plasma might-be observable in the periods when solar clouds were observable. Were any useful observations of those clouds made?

DR. BIERMANN: I am not aware of observations of plasma clouds. I have heard only of observations of artificial natrium clouds.

QUESTION: Sodium clouds?

DR. BIERMANN: Yes. But the observed clouds are not ionized. When ionized, sodium becomes invisible. So what is observed is the nonionized component, and that is analogous to the $\mathrm{CN}$ and $\mathrm{C}_{2}$, but not to the plasma. The nonionized component doesn't give you any information about the solar plasma. It is simply acted on by solar light. Whatever influence the plasma can exert on the nonionized component would be quite small compared with the light pressure.

QUESTION: If the ionization of $\mathrm{CO}$ and $\mathrm{N}_{2}$ occur through charge exchange, is there any reason why $\mathrm{C}_{2}$ and $\mathrm{CN}$ cannot be ionized in the same way? $\mathrm{CN}$, I think, would be visible-much better than $\mathrm{C}_{2} . \mathrm{C}_{2}$, I think, wouldn't be visible in the low-lying state.

DR. BIERMANN: In this particular case I was rather thinking of photoionization, and not of ionization by exchange of charge. Of course, it might very well be that charge exchange also enters significantly. That I don't know. But the cross section for exchange of charge depends in a fairly complicated way on the properties of the spectrum. It seems that the fact that both $\mathrm{CO}^{+}$and $\mathrm{N}_{2}{ }^{+}$ have low-lying levels giving rise to the observable resonance terms may have some connection with the fact that the cross section for exchange of charge is large-in the velocity range of a few hundred to a few thousand kilometers per second [see the work by Gilbody and Hasted where further references may be found; Proc. Roy. Soc. A 238:334(1956)].

I am not aware of any resonance lines of nonionized $\mathrm{CO}$ in the optical range. I was also under the impression that the ion of $\mathrm{CN}$ does not have any observable resonance bands. If I am wrong there, then of course it would be very significant in connection with the questions you are discussing.

QUESTION: You mentioned the fact that there is a rotation observed in tails sometimes. Is this possibly explainable in terms of a longitudinal drift of particles in a field gradient? 
DR. BIERMANN: The way we tend to look at it is this: You know perhaps of the work of Lüst and Schlüter on force-free fields. First, Lundquist, then Lüst and Schlüter, and later still others discussed the properties of force-free fields. One of the main properties is that they have a sort of spiral structure if there is any symmetry at all.

Now, we do observe occasionally a feature that I have not mentioned, something like a largescale helical structure in the tail (Type I) of a comet. The tail then looks like a large helix viewed from the side. Of course, that is not an unambiguous interpretation, but it is one that we would naturally think of. We have liked this idea, even since we became aware of the probability that there were magnetic fields that should be force-free.

Of course, the situation might be more complicated than that.

QUESTION: As an extension of this, is there any information about whether there is a hole in the magnetic field behind the comet? Whether the solar field is excluded by the comet?

DR. BIERMANN: There is no information concerning the magnetic field; but there is an indication, which I had not mentioned, as the particle stream. The indication is this: If the acceleration is measured as a function of position in the tail, it is often found (e.g., in the work of Peter Stumpff on comet Morehouse and in that of Rhea Lüst on comet Mrkos) that the acceleration strongly decreases, possibly to about zero in the vicintiy of the tail axis at some distance behind the coma.

So it looked as though in these cases the influence of the interaction of the solar corpuscular radiation with the comet's coma was of such a kind that the immediate vicinity of the axis of the tail was excluded from further impact of solar corpuscular radiation. 